\title{
The Thermal Pressure of the Interstellar Medium Derived from Cloud Shadows in the Extreme Ultraviolet
}

\author{
Thomas W.Berghöfer ${ }^{1}$, Stuart Bowyer ${ }^{1}$, Richard Lieu ${ }^{2}$, and Jens Knude ${ }^{3}$ \\ 1 Space Sciences Laboratory, University of California, Berkeley, CA 94720, USA \\ ${ }^{2}$ Department of Physics, University of Alabama, Huntsville, USA \\ 3 Niels Bohr Institute for Astronomy, Physics, and Geophysics, University Obser- \\ vatory, Juliane Mariesvej 30, DK-2100 Copenhagen OE, Denmark
}

\begin{abstract}
We have used the Deep Survey telescope of EUVE to investigate shadows in the diffuse EUV/Soft X-ray background cast by clouds in the interstellar medium. We confirm the existence of a shadow previously reported, and provide evidence for two new shadows. We used IRAS data to identify the clouds producing these shadows and to determine their optical depth to EUV radiation. The EUVabsorbing clouds are optically thick in the EUV, and all EUV emission detected in the direction of these shadows must be produced from material in front of the clouds. We obtained new optical data to determine the distance to these clouds. We use a new differential cloud technique to obtain the pressure of the interstellar medium. These results do not depend on any zero level calibration of the data. Our results provide evidence that the pressure of the hot interstellar gas is the same in three different directions in the local interstellar medium, and is at least 8 times higher than derived for the local cloud surrounding our Sun. This provides new evidence for large thermal pressure imbalances in the local ISM, and directly contradicts the basic assumption of thermal pressure equilibrium used in almost all present models of the interstellar medium.
\end{abstract}

\section{Cloud Shadow Observations with EUVE}

Prior to this paper, Bowyer et al. (1995) reported on the first detection of a spatial absorption feature in the diffuse EUV background; this feature discovered in the EUVE Deep Survey (DS) was positionally coincident with an IRAS cirrus cloud at a distance of $\lesssim 40$ pc. By attributing the background subtracted flux in front of the cloud to the hot gas in the ISM, Bowyer et al. (1995) derived a pressure $(\mathrm{P} / k)$ of $19,000 \mathrm{~cm}^{-3} \mathrm{~K}$. Here we discuss a reobservation of this initial shadow and we present data for two new cloud shadows discovered in the EUV background. These shadows are located near ecliptic longitudes $\lambda=52^{\circ}$ (initial shadow), $\lambda=309^{\circ}$, and $\lambda=214.5^{\circ}$; according to the galactic coordinates of these cloud locations they are denoted as $\mathrm{lb} 165-32,1 b 27-31$, and $\mathrm{lb} 329+46$ in this paper. A recent re-observation of the initially discovered cloud shadow lb165-27 confirmed the existence of this cloud shadow. In both the original DS scan as well as in the re-observation, 
the $(3 \sigma)$ absorption feature in the EUV background is positionally coincident with an enhanced emission feature in the IRAS $100 \mu \mathrm{m}$ data. Exemplary for the three cloud shadows, Fig. 1 provides the EUVE DS observations of 1b27-31.

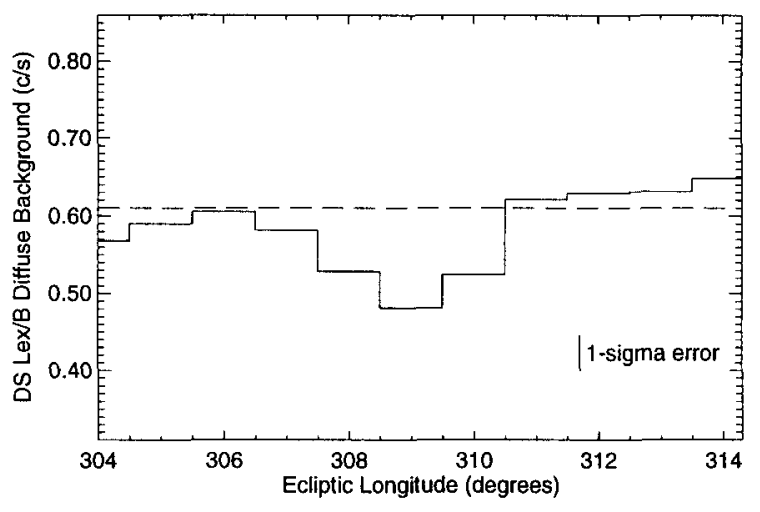

Fig. 1. EUV diffuse background count rate in the Lex/B filter as a function of ecliptic longitude

We have used detailed Strömgren photometry of stars in the cloud shadow regions to determine the distance to the EUV-absorbing clouds. The distances of the EUV-shadowing clouds have been constrained by finding the most distant unreddened star in front of a cloud and the closest reddened star behind this cloud. This method was also applied to determine the distance where optical depth reaches unity in the regions immediately adjacent to the shadowing cloud. For all three cloud shadows our optical data provide evidence for a second larger cloud ("background cloud") located behind the nearby EUV-absorbing cloud.

In Fig. 2 we plot the DS Lex/B filter count rates versus the respective distances for all three cloud shadows. For all shadows Fig. 2 shows the count rates and distances obtained at the position of the shadow as well as in front of the background cloud. For $1 \mathrm{~b} 329+46$ a third data is included that originates from a scan region showing a much larger emission column along the line of sight. The different cloud shadow regions are marked by different symbols, the distance upper limits are indicated by left arrows. As is evident from Figure 2 almost all the data points are reasonably fit with a straight line. The formal results of a linear regression analysis of the data are $0.002 \pm$ $0.0003 \mathrm{cts} / \mathrm{s} \mathrm{pc}^{-1}$ for the slope and $0.331 \pm 0.045 \mathrm{cts} / \mathrm{s}$ for the intercept of the regression line. This line is plotted in Figure 2. The slope of the regression line gives the diffuse EUV flux per emitting length which we use to derive the pressure of the plasma in the ISM. 


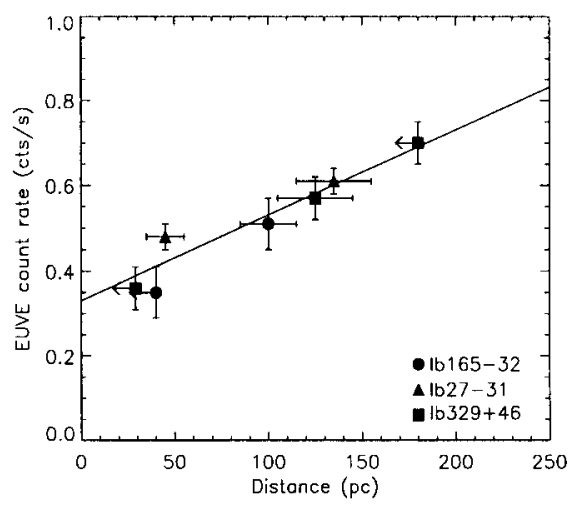

Fig. 2. Plot of all observed count rates versus the distance of the respective clouds; distance upper limits are indicated by left arrows. The solid straight line provides the results of a linear regression analysis.

\section{The Thermal Pressure of the ISM}

We now utilize the results of our cloud shadow observations to compute the pressure of the local ISM from the ideal gas law. For the conversion of the observed EUVE DS count rates per emitting length into EM/L we use the typically assumed model spectrum of a plasma in collisional ionization equilibrium at a temperature of $10^{6} \mathrm{~K}$. To account for the absorption of the local cloud around our Sun we include a foreground ISM absorption of $\mathrm{N}_{\mathrm{H}}=5 \times 10^{18} \mathrm{~cm}^{-2}$ in our model. With the plasma emissivity code provided by Landini \& Monsignori-Fossi we derive $\mathrm{EM} / \mathrm{L}=7.3 \times 10^{-5} \mathrm{~cm}^{-6}$. The pressure of the hot ISM is thus $\mathrm{P} / k \approx 16500 \mathrm{~cm}^{-3} \mathrm{~K}$. We mention that for a Raymond \& Smith model of the same parameters we obtain $\mathrm{EM} / \mathrm{L}=$ $5.1 \times 10^{-5} \mathrm{~cm}^{-6}$ and $\mathrm{P} / k \approx 13500 \mathrm{~cm}^{-3} \mathrm{~K}$. This value is close to pressure values for the "Local Bubble" published by other authors in these proceedings.

The canonical value obtained for the local cloud is $\mathrm{P} / k=730 \pm 30 \mathrm{~cm}^{-3} \mathrm{~K}$ (e.g., Frisch 1995). Based on line measurements with EUVE, Vallerga (1996) derived a pressure for the local cloud in the range $\mathrm{P} / k=1700-2300 \mathrm{~cm}^{-3} \mathrm{~K}$. A comparison of either of these results with the pressure we obtained for the hot ISM shows the pressure of the local ISM exceeds the pressure of the local cloud surrounding our Sun by a factor of $\geq 8$. The tight correlation between observed diffuse EUV flux and emitting length implies the same pressure in three different cloud directions (cf. Fig. 3). The original observation (Bowyer et al. 1995) of a pressure imbalance between the local solar cloud and the surrounding ISM could have been the result of a nearby shock wave which had not yet reached the Sun. The isotropy in the results for all these directions is not consistent with this hypothesis. The zero level calibration for our cloud shadow observations with the EUVE DS instrument 


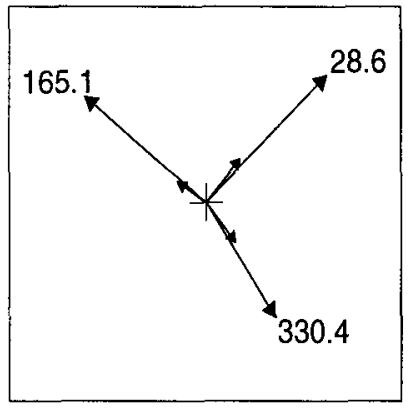

Fig. 3. Projection of the sky directions towards the cloud shadows as seen from above the Galactic plane; numbers give the Galactic longitude, the length of the arrows scales with the projected distance of the clouds.

allows us to measure absolute fluxes for the diffuse EUV background. These zero level corrected values also give the same pressure for the material in the foreground of the three shadowing clouds.

In summary, our observations provide evidence for a large pressure imbalance in the local ISM compared to the local cloud surrounding our Sun. This contradicts the basic assumption of almost all available models for the local ISM of a pressure equilibrium (e.g., McKee \& Ostriker 1977). It is unclear what physical mechanism can maintain such a large pressure imbalance. We emphasize that these models as well as the calculations in the here presented work assume a hot plasma in collisional ionization equilibrium. A completely different approach is provided by Breitschwerdt \& Schmutzler (1994) . Their model calculations are based on an adiabatically cooling non-equilibrium plasma. The advantage of this model is that it can at least qualitatively explain many of the observed features of the local ISM and it does not require pressure equilibrium in the local ISM.

Acknowledgements. We thank M. Lampton and J. Vallerga for useful discussions. T.W.B. acknowledges the support from the Alexander-von-Humboldt Stiftung $(\mathrm{AvH})$ by a Feodor-Lynen Fellowship. This work has been supported by NASA contract NAS 5-30180. R.L. is supported by NASA grant 5-34378 awarded to UAH. J.K. thanks the European Southern Observatory for observing time and travel support.

\section{References}

Bowyer, S., Lieu, R., Sidher, S. D., Lampton, M., Knude, J. 1995, Nature, 375, 212 Breitschwerdt, D., Schmutzler, T., 1994, Nature, 371, 774

Frisch P. C., 1995, Space Sci. Rev., 72, 499

McKee, C. F., Ostriker, J. P., 1977, ApJ, 218, 148

Vallerga, J., 1996, Space Sci. Rev., 78, 277 